\title{
Dengue Virus Cases Surge Amidst COVID-19 in Pakistan: Challenges, Efforts and Recommendations
}

\author{
Zarmina Islam', Parvathy Mohanan², Wajeeha Bilal', Taleen Hashmi', Zainab Rahmat', Ikran Abdi', \\ Mehr Muhammad Adeel Riaz ${ }^{4}$, Mohammad Yasir Essar (iD ${ }^{5}$ \\ 'Faculty of Medicine, Dow Medical College, Dow University of Health Sciences, Karachi, Pakistan; ${ }^{2}$ Medical University Sofia, Sofia, Bulgaria; ${ }^{3}$ Faculty of \\ Medicine, Jinnah Sindh Medical University, Karachi, Pakistan; ${ }^{4}$ Punjab Medical College, Faisalabad, Pakistan; ${ }^{5}$ Kabul University of Medical Sciences, \\ Kabul, Afghanistan
}

Correspondence: Mohammad Yasir Essar, Kabul University of Medical Sciences, Kabul, Afghanistan, Tel +992 II7I I8300, Email Yasir.essar@gmail.com

\begin{abstract}
Pakistan is currently facing two outbreaks, dengue and COVID-19; both have strained its healthcare system resulting in multiple concerns including the co-diagnosis of two. Due to poor healthcare capacity, low vaccination rate, increasing COVID-19 variants, socioeconomic disparities, and misinformation, it is inevitable that implications will prove to be damaging to both healthcare workers and civilians. Among these challenges, it is important to note the need for stronger epidemiological surveillance for both COVID-19 and dengue and the implementation of public health measures without endangering sources of livelihood. To sustain this, cooperation between WHO and Pakistan's government must continue through smart lockdowns, dengue awareness campaigns, and double laboratory procedures.
\end{abstract}

Keywords: dengue, COVID-19, Pakistan, healthcare infrastructure

\section{Introduction}

Dengue is a mosquito-borne viral infection typically seen in tropical and subtropical regions. It has four different strains (DENV-1, DENV-2, DENV-3, and DENV-4), all belonging to the Flaviviridae family. ${ }^{1}$ The infection transmits through bites of the infected female Aedes aegypti or Aedes albopictus mosquito that has previously been infected by biting a person with the dengue virus. ${ }^{1}$ In 1994, Pakistan reported the first confirmed outbreak of dengue fever. However, the annual epidemic trend and unexpected rise in cases first became apparent in November 2005. ${ }^{2,3}$ Since 2010, Pakistan has reported tremendous numbers of dengue cases, with 2020 alone amounting to 47,120 confirmed cases including 75 deaths $^{4}$. As of 25 November 2021, a total of 48,906 cases of Dengue including 183 deaths have been reported. ${ }^{5}$ Unfortunately, this debilitating, mosquito-borne disease is now endemic in Pakistan circulating throughout the year with the highest prevalence in the post-monsoon season with all four provinces being majorly affected. ${ }^{3}$ The rapid spread of the infection is attributed to irregular monsoon rains and rising temperatures linked to climate change. Such favorable factors allow for ideal mosquito breeding conditions thus aids virus survival. ${ }^{6}$ Adverse circumstances including inadequate sanitation and garbage disposal, unsafe drinking water, overcrowded cities, and breakneck urbanization, lack of vector control strategy, and the clinical management of hospitalized dengue cases have promoted the transmission of the infectious agent. ${ }^{1}$ On October 2nd, 2021, Pakistan documented 1664 COVID-19 cases and 46 deaths over the span of 24 hours. ${ }^{7}$ The number of active COVID-19 cases has plummeted to 46,934 with a high recovery rate, the lowest it has been in the past two months. ${ }^{7}$ Pakistan has administered a total of $82,830,350$ COVID-19 vaccines, vaccinating about $19.1 \%$ of the country's population as of October $2021 .^{7}$ Since 2020 , studies have reported numerous cases of a new coinfection of COVID-19 and dengue that has a higher mortality rate than patients only diagnosed with COVID-19. ${ }^{5}$ The two viruses have been shown to have overlapping clinical and laboratory features. Both are commonly characterized by fever, severe headache (clinical presentation), thrombocytopenia and leukocytopenia (on lab findings). The coinfected group has also observed high percentages of bilirubin, alanine amino transferase, creatinine, prothrombin time, urea, and 
creatinine phosphokinase. ${ }^{5}$ From June 23rd through 15 th July 2021, 20 suspected patients of COVID-19 with respiratory distress, were admitted to Holy Family Hospital in Rawalpindi. Upon collection of respiratory swabs, it was found that all 20 patients tested positive for SARS-CoV-2 and dengue virus serotype-2 (DENV-2). ${ }^{8}$ The province of Punjab has reported a total of 1082 cases of dengue fever this year with Lahore at high risk of facing a major dengue outbreak following the recent rainfalls. ${ }^{9,10}$ This has led to serious public health crises due to the absence of active surveillance and diversion of entire efforts towards the fight against COVID-19, lack of lockdown regulations, meager implementation of preventive measures, paucity of knowledge in the public, and mass gatherings in worship places. ${ }^{11,12}$

\section{Challenges and Efforts}

Just as nations like Saudi Arabia, Brazil, Yemen, Sudan, and Madagascar are presently confronting episodes of dengue with COVID-19, so is Pakistan. ${ }^{13,14}$ Significant similarities in the haematological and biochemical markers like fever, headache, and body pain, and laboratory characteristics like thrombocytopenia and leukopenia between the dengue and COVID-19 infected patients are of concern, especially for Pakistan's ill-equipped healthcare infrastructure. ${ }^{4}$ The coepidemic of COVID-19 and dengue in Pakistan has brought about numerous public health challenges on the delicate healthcare framework of the nation. Moreover, since dengue infection (DENV) transmission is strongly driven by human mobility, changes associated with the pandemic have been hypothesized to impact dengue incidence. By debilitating human contact, COVID-19 control measures have also disturbed dengue vector control medications, which are the most effective way of mitigating the disease. ${ }^{15}$

Pakistan's provincial healthcare framework has been terribly neglected due to political interference, with a weak administrative system. Most imperatively, all vertical programs are run by the Federal government without the coordination of the Common Government. ${ }^{4,16}$ This disregard has resulted in enormous disparities between getting access to basic healthcare facilities for both the poor and wealthy. ${ }^{17}$ There is also much less information accessible in Pakistan on the prevalence and clinical and laboratory characteristics of dengue, and hence complications in epidemiological surveillance. $^{2}$ This coupled with a colossal burden on finances within the country complicates vector control measures. Moreover, limitations in essential resources for healthcare professionals (HCPs) battling COVID-19 and other endemic illnesses like dengue and chikungunya, as well as COVID-19-Associated Mucormycosis (CAM) in severely ill COVID19 patients will inevitably add to both mortality and morbidity. ${ }^{18,19}$ Lastly, maintaining social distancing in urban centres like Karachi and Lahore is difficult, and stigma around COVID-19 vaccinations further creates vulnerabilities that may impose serious strain on healthcare infrastructure. ${ }^{20}$ Furthermore, a reliance on unsustainable sources of income and high levels of poverty are adding to higher mobility, reducing implementation of quarantine, and hence contributing to both Dengue and COVID-19. ${ }^{4,21}$ The implications of such constrained resources with poor distancing measures exacerbates and prolongs both the development and implementation of healthcare measures. However, despite socioeconomic disparities, economic failure and difficulties with public health measures, Pakistan continues to be persistent in its plans to address Dengue and COVID-19.

In response to the dengue outbreak and to assist with vector control, WHO entomologists studied the Aedes mosquito in Karachi during the 2005 outbreak and used this information to design Pakistan-specific control interventions. ${ }^{2}$ In the past outbreaks, chemical and environmental management techniques have been used to curb the spread of dengue including fumigation and spraying, improving solid waste disposal, and applying insecticides to larval habitats. Alternatively, using the media to promote healthy practices such as drinking large amounts of fluids, rest, complete blood count (CBC) if fever persists beyond 3 days, intake of Oral rehydration salt (ORS), and keeping body temperature below $39 \mathrm{C}$. Community-based programs, provision of long-term insecticides, insecticide treated nets (ITNs) to vulnerable groups such as children and pregnant woman, and involvement of provincial and municipal health authorities in surveillance and policies have proven helpful. ${ }^{3}$ Currently, the WHO country office (WCO) is supporting the government of Pakistan in the following ways: ${ }^{22}$ Developing a comprehensive dengue response plan covering case management, dengue surveillance, vector control, and community mobilization and coordination along with donation of mosquito fogging equipment with thousands of dengue awareness flyers, posters, banners and brochures, which were distributed in schools, railway stations and other public places to provide information on the dengue virus. ${ }^{22}$ Response measures taken by the Ministry of Health in Pakistan include the formation of The National Institute of Health 
Emergency Operation Center (NIH EOC) since 16 September 2019 to monitor the disease situation in constant liaison with the provincial health departments. ${ }^{22}$ In addition, rapid response teams are in place to conduct active response at household level and in infected areas and all provinces have arranged free of charge diagnostic and clinical management services for the cases. ${ }^{22}$ At the moment, there is no suitable treatment available for the dengue fever caused by this virus or an effective vaccine, and clinicians can only halt the progression of the viral infection to its hemorrhagic state. $^{23}$

\section{Recommendations and Conclusion}

Exceptional measures should be taken like large investments and research in epidemiological, diagnostics, and vaccine advancement research, therapeutic and protective supplies are required to tackle the epidemic productively. Careful surveillance, viral identification tests, and disinfecting large areas should be prioritized to identify and restrain the transmission of viruses. ${ }^{11}$ Moreover, employing a multisectoral approach involving provincial and municipal healthcare authorities to enforce strict public health measures such as checking body temperature in public spaces, encouraging no touch delivery services, and social distancing is recommended. Preventive measures that individuals should adopt include using insect repellent, keeping bodies covered by wearing long sleeved shirts and pants, drinking clean water, using insecticide sprays in dark corners of your home, covering all the water storage containers, and storing them.

Complete blood count $(\mathrm{CBC})$ is the single most common test performed in patients. In dengue, the $\mathrm{CBC}$ is characterized by progressive leukopenia followed by a rapid decrease in the platelet count, while in contrast, patients with COVID-19 may present with leukopenia or leukocytosis, eosinopenia, and lymphopenia which is the most common abnormality, hence makes it a useful and reliable indicator of the severity of the disease. To add, it is also cost-effective and thus feasible to implement in Pakistan. Other than that, double laboratory testing will be a more accurate source of diagnosis differentiating the two. DEN-PCR is successful for diagnosis in Pakistan for both primary and secondary dengue infections. ${ }^{24}$ Although serum antibody test may be used for differential diagnosis, HCPs must be careful to avoid false positive cases. ${ }^{25}$ Donation of mosquito fogging equipment, along with dengue awareness flyers, posters, banners, and brochures, which can be distributed in public places to provide information on the Dengue virus is also recommended. Lastly, the continual use of smart lockdown in vulnerable areas of the city to address COVID-19 can help reduce economic strain on those with unsustainable incomes, whereas travel restrictions within the city for unvaccinated individuals increase the rate of vaccination. More importantly, efforts such as counseling services and a mental health hotline are to be considered for HCPs who face high levels of burnout. ${ }^{26-28}$

In conclusion, Pakistan is currently facing multiple outbreaks of Dengue and COVID-19 that have exacerbated challenges for a weak healthcare system. Coupled with socioeconomic disparities, financial challenges, a slow vaccination rate, multiple clinical similarities, weak social distancing and increasing workload for HCPs, it is becoming difficult to mitigate these outbreaks. However, continual efforts by the WHO as well as preventative measures and epidemiological surveillance will aid this process.

\section{Abbreviations}

COVID-19, coronavirus disease 2019; HCP, healthcare professionals.

\section{Consent for Publication}

All authors agreed to the publication of this manuscript.

\section{Author Contributions}

All authors contributed to data analysis, drafting or revising the article, have agreed on the journal to which the article was submitted, gave final approval of the version to be published, and agreed to be accountable for all aspects of the work. 


\section{Funding}

No funding was received for this work.

\section{Disclosure}

The authors declare that they have no conflicts of interest for this work.

\section{References}

1. IAMAT. Pakistan: dengue. Available from: https://www.iamat.org/country/pakistan/risk/dengue. Accessed October 3, 2021.

2. WHO EMRO. Dengue fever | programmes | Pakistan. Available from: http://www.emro.who.int/pak/programmes/dengue-fever.html. Accessed October 3, 2021.

3. Jahan F. Dengue Fever (DF) in Pakistan. Asia Pac Fam Med. 2011;10(1):1. doi:10.1186/1447-056X-10-1

4. Suleman rana M, Usman M, Masroor Alam M, Ikram A, Salman M. Overlapping clinical manifestations of COVID-19 with endemic infectious diseases in Pakistan: a looming threat of multiple lethal combinations. Infect Ecol Epidemiol. 2021;11(1):1873494.

5. Dengue fever - Pakistan. Available from: https://www.who.int/emergencies/disease-outbreak-news/item/dengue-fever-pakistan. Accessed January 7, 2022.

6. Record-high 44,000 dengue cases reported in Pakistan | Health News | Al Jazeera. Available from: https://www.aljazeera.com/news/2019/11/6/ record-high-44000-dengue-cases-reported-in-pakistan. Accessed October 3, 2021.

7. COVID-19 in Pakistan kills 46 people in last 24 hours. Available from: https://www.geo.tv/latest/373543-covid-19-in-pakistan-kills-46-people-inlast-24-hours. Accessed October 5, 2021.

8. Saddique A, Suleman Rana M, Masroor Alam M, et al. Emergence of co-infection of COVID-19 and dengue: a serious public health threat. J Infect. 2020;81(6):e16. doi:10.1016/j.jinf.2020.08.009

9. Pakistan reports surge in dengue fever cases amid outbreak fears - Xinhua. Available from: http://www.news.cn/english/2021-09/26/c_1310211119. $\mathrm{htm}$. Accessed October 3, 2021.

10. Rising dengue cases - newspaper. DAWN.COM. Available from: https://www.dawn.com/news/1648071. Accessed October 3, 2021.

11. Rana MS, Alam MM, Ikram A, Zaidi SSZ, Salman M, Khurshid A. Cocirculation of COVID-19 and dengue: a perspective from Pakistan. J Med Virol. 2021;93(3):1217-1218. doi:10.1002/JMV.26567

12. Coronavirus command center reports people not following SOPs - Pakistani planning minister. Available from: https://www.arabnews.pk/node/ 1717916/pakistan. Accessed October 3, 2021.

13. Rabiu AT, Mohan A, Çavdaroğlu S, et al. Dengue and COVID-19: a double burden to Brazil. J Med Virol. 2021;93(7):4092. doi:10.1002/ JMV.26955

14. Raza FA, Javed H, Khan MM, et al. Dengue and Chikungunya virus co-infection in major metropolitan cities of provinces of Punjab and Khyber Pakhtunkhwa: a multi-center study. PLoS Negl Trop Dis. 2021;15(9):e0009802. doi:10.1371/JOURNAL.PNTD.0009802

15. Tsheten T, Clements ACA, Gray DJ, Adhikary RK, Wangdi K. Clinical features and outcomes of COVID-19 and dengue co-infection: a systematic review. BMC Infect Dis. 2021;21(1):1-9. doi:10.1186/S12879-021-06409-9

16. Zaidi SA, Bigdeli M, Langlois EV, et al. Health systems changes after decentralisation: progress, challenges and dynamics in Pakistan. BMJ Glob Heal. 2019;4(1). doi:10.1136/BMJGH-2018-001013

17. Yusufzai A. WHO drafted in to help control severe dengue outbreak in Pakistan. BMJ. 2019;367:16083. doi:10.1136/BMJ.L6083

18. Shakoor MT, Ayub S, Ayub Z. Dengue fever: Pakistan's worst nightmare. WHO South-East Asia J Public Health. 2012;1(3):229-231. doi:10.4103/ 2224-3151.207018

19. Riad A, Shabaan AA, Issa J, et al. COVID-19-Associated Mucormycosis (CAM): case-series and global analysis of mortality risk factors. $J$ Fungi. 2021;7(10):837. doi:10.3390/JOF7100837

20. Social distancing in Pakistan: on JSTOR. Available from: https://www.jstor.org/stable/resrep29098?seq=1\#metadata_info_tab_contents. Accessed October 3, 2021.

21. Khalid A, Ali S. COVID-19 and its challenges for the healthcare system in Pakistan. Asian Bioeth Rev. 2020;12(4):551. doi:10.1007/S41649-02000139-X

22. World Health Organization. Outbreak update - Dengue in Pakistan, 19 November 2019. ReliefWeb; 2019. Available from: https://reliefweb.int/ report/pakistan/outbreak-update-dengue-pakistan-19-november-2019. Accessed October 3, 2021.

23. International Federation of Red Cross and Red Crescent Societies. Pakistan: Dengue Outbreak Emergency Plan of Action (EPoA) DREF Operation $\mathrm{n}^{\circ}$ MDRPK017 - Final Report. ReliefWeb; 2020. Available from: https://reliefweb.int/report/pakistan/pakistan-dengue-outbreak-emergency-planaction-epoa-dref-operation-n-mdrpk017-final. Accessed October 3, 2021.

24. Hasan Z, Razzak S, Farhan M, et al. Increasing usage of rapid diagnostics for Dengue virus detection in Pakistan. J Pak Med Assoc. 2017;67 (4):548-551.

25. Santoso MS, Masyeni S, Haryanto S, Yohan B, Hibberd ML, Sasmono RT. Assessment of dengue and COVID-19 antibody rapid diagnostic tests cross-reactivity in Indonesia. Virol J. 2021;18(1):1-5. doi:10.1186/S12985-021-01522-2/TABLES/3

26. Islam Z, Gangat SA, Mohanan P, et al. Mental health impacts of Lebanon's economic crisis on healthcare workers amidst COVID-19. Int J Health Plann Manage. 2021:hpm.3324. doi:10.1002/HPM.3324.

27. Islam Z, Rocha ICN, Mohanan P, et al. Mental health impacts of humanitarian crisis on healthcare workers in Yemen. Med Confl Surviv. 2021;37 (2):112-117. doi:10.1080/13623699.2021.1950519

28. Essar MY, Hasan MM, Islam Z, Riaz MMA, Aborode AT, Ahmad S. COVID-19 and multiple crises in Afghanistan: an urgent battle. Confl Health. 2021;15(1):1-3. doi:10.1186/S13031-021-00406-0 


\section{Publish your work in this journal}

Infection and Drug Resistance is an international, peer-reviewed open-access journal that focuses on the optimal treatment of infection (bacterial, fungal and viral) and the development and institution of preventive strategies to minimize the development and spread of resistance. The journal is specifically concerned with the epidemiology of antibiotic resistance and the mechanisms of resistance development and diffusion in both hospitals and the community. The manuscript management system is completely online and includes a very quick and fair peer-review system, which is all easy to use. Visit http://www.dovepress.com/testimonials.php to read real quotes from published authors.

Submit your manuscript here: https://www.dovepress.com/infection-and-drug-resistance-journal 